\title{
Redes de influência no Congresso \\ Nacional: como se articulam os \\ principais grupos de interesse
}

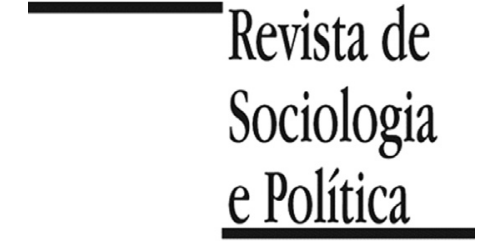

DOI 10.1590/1678-987316245906

\author{
Pablo Silva Cesário
}

\section{Resumo}

O objetivo do artigo é mapear as relações entre grupos de interesse no Congresso Nacional brasileiro e levantar hipóteses sobre a população de grupos de interesse. As perguntas que norteiam este trabalho são: como os grupos de interesse se articulam ao redor das principais discussões políticas? Quais são os grupos mais ativos? Quais são as principais características dessa estrutura de relações? Os dados para a construção da rede de grupos de interesse foram coletados a partir das páginas da Câmara dos Deputados e do Senado Nacional. A pesquisa buscou registrar todas as participações em audiências públicas e seminários nos anos de 2011 e 2012 , período a partir do qual se encontrou melhor qualidade nos relatórios. Trata-se, portanto, de uma rede completa, com foco multitemático e de todas as comissões do Congresso Nacional, permanentes ou não. A rede completa de grupos de interesse é de baixa densidade de uma forma geral e caracterizada pela formação de clusters muito bem definidos ao redor de políticas públicas específicas, com muitas organizações especializadas em poucas políticas públicas. Do ponto de vista de influência, existe um subconjunto de grupos de interesses que possui relações mais intensas entre si e com os demais que pode ser chamado de núcleo e pode, portanto, ser identificado como aqueles de maior influência. Todavia, apesar da assimetria, nenhum grupo de interesse ou tipo de grupo de interesse está sequer perto de exercer a hegemonia dada a baixa centralização da rede e até mesmo a variância dos graus de influência. Os resultados são a primeira análise completa de uma rede de grupos de interesse no mundo. Eles apontam para a existência de um núcleo diversificado de grupos de interesse que são mais capazes de exercer a influência, o que indica a necessidade de revisão da pesquisa na área para dar conta dessa natureza distinta. Do ponto de vista teórico, apresenta um indício de que a tese pluralista de atomização de grupos de interesse deve ser reavaliada.

PALAVRAS-CHAVE: grupos de interesse; Congresso Nacional; análise de redes; lobby; processo decisório.

Recebido em 2 de Maio de 2015. Aceito em 8 de Julho de 2015.

\section{Introdução ${ }^{1}$}

${ }^{1}$ Agradecemos aos comentários e sugestões dos pareceristas anônimos da Revista de Sociologia e Política.
$\mathrm{C}$ omo parte do mesmo ambiente político, grupos de interesse possuem relações de cooperação ou competição entre si. Formam, assim, uma ampla rede que liga desde os movimentos locais até as organizações nacionais que funcionam como ponta de lança de interlocução com autoridades políticas, ou mesmo orgãos internacionais. O objetivo deste artigo é mapear as relações entre grupos de interesse no Congresso Nacional brasileiro e levantar hipóteses sobre a população de grupos de interesse. As perguntas que norteiam este trabalho são: como os grupos de interesse se articulam ao redor das principais discussões políticas? Quais são os grupos mais ativos? Quais são as principais características dessa estrutura de relações?

O método escolhido foi a análise de redes porque que permite uma investigação baseada nas relações entre grupos de interesse e oferece indicadores para descrevê-los. Ainda, permite uma nova perspectiva sobre uma questão antiga, mas ainda pendente, sobre a influência dos grupos de interesse. Uma literatura crescente tem equacionado a influência de agentes sociais com o padrão de inserção na rede de relacionamentos. Em geral, a tese é que a centralidade de um agente em uma determinada rede está relacionada com o poder ou influência 
desse agente. Aplicado aos grupos de interesse, propõe-se a hipótese de que a "influência" política pode ser medida a partir do posicionamento de um determinado grupo de interesse na rede de acesso a instâncias decisórias. Propõe-se, desse modo, que a influência também pode ser medida a partir das relações sociais e não apenas das "fontes de poder" ou da efetividade de suas iniciativas de defesa e promoção de interesses, tal como tem sido mais frequente na literatura.

Assim, outras perguntas enfrentadas são: qual é a centralidade de cada um desses grupos de interesse? Como a centralidade é distribuída entres os grupos de interesse? Existe uma assimetria fundamental em favor de tipos de grupos de interesses?

\section{Influência em redes}

O estudo de redes em Ciência Política tem passado por um momento de grande produtividade, tal como demonstram Ward, Stovel e Sacks (2011) e Lazer (2011). A razão para esse entusiasmo é que a análise dos relacionamentos de agentes políticos (indivíduos, organizações ou estados) permite novos olhares. Em primeiro lugar, a incorporação os padrões de relações permite analisar as interdependências entre agentes. Por exemplo, é possível incorporar as influências mútuas até mesmo na formação dos interesses de grupos de pressão. Em segundo lugar, a partir da posição de um agente político em uma rede, fica clara a estrutura de oportunidades e constrangimentos que ele enfrenta (Huckfeldt 2009), especialmente no que toca aos custos de transação.

Lazer (2011) aponta que umas das áreas mais promissoras de redes em Ciência Política é o estudo sobre os efeitos de rede. Muito frequentemente a investigação tem estado voltada para o entendimento da rede como uma estrutura de circulação, de informação, adoção de inovações, agentes patogênicos, influência social (convergência de comportamento e atitudes) etc. Assim, redes de grupos de interesse, por onde circulam informações e influência, podem ter efeitos políticos distintos se apresentarem estruturas distintas e grupos podem ter capacidades diferenciadas de acordo com o padrão de inserção na rede.

Borgatti et al. (2009) levam o argumento mais adiante ao defenderem que a estrutura e a posição de cada nó na rede podem ser um preditor importante de desempenho. Por exemplo, empresas de maior centralidade demonstram melhor desempenho em inovação por número de patentes e também melhores resultados financeiros. Outras pesquisas têm indicado que indivíduos com maior centralidade são mais influentes. Degenne e Forsé (1994) vão ainda mais longe e defendem que "power and prestigie are measured by determining how actors with special goals and interests exchange resources for a variety of events to their own advantage". Na Sociologia, uma referência sobre a relação entre posição na rede e influência social pode ser vista em Marsden e Friedkin (1993).

Aqui está, portanto, uma questão importante para o estudo de redes de grupos de interesse: seus papeis estruturais podem resultar em maior capacidade de exercer influência? Poderia a centralidade ser utilizada como uma proxy para influência (ou poder)?

Diversos estudos sobre redes que envolvem grupos de interesse têm sido publicados. Os principais temas têm sido financiamento de campanhas parlamentares, redes de políticas públicas específicas, relações, percepção de influência em redes multiplex, tribunais superiores e estabilidade de redes de grupos de interesse.

Pappi e Henning (1998; 1999) e Henning (2009) buscaram explorar a relação entre grupos de interesse e autoridades governamentais. A proposição 
teórica é que a rede de grupos de interesse e agentes governamentais funciona como uma estrutura de troca de recursos de influência, tais como conhecimento técnico, monitoramento de informações, apoio público por parte de grupos de interesse e controle sobre as decisões políticas dos agentes governamentais. Neste caso, redes tendem a afetar os resultados de políticas públicas porque nem todas as relações que poderiam ser estabelecidas entre os agentes da rede seriam mutuamente benéficas, e se efetivam na medida em que há custos de transação. Assim, a rede pré-existente condiciona as escolhas políticas possíveis e afeta os resultados. Em outros termos, no nível macro, a rede funciona como um mecanismo de facilitação de trocas políticas e, no nível micro, a posição de cada um dos agentes pode em si mesma se tornar um elemento de influência.

Na tentativa de realizar testes empíricos, Pappi e Henning (1999) e Henning (2009) mapearam a rede grupos de interesse atuando sobre a política agrícola europeia. A estratégia de seleção se baseou em entrevistas sobre a percepção de influência de grupos regionais e nacionais feitas a grupos de interesses e autoridades políticas. $\mathrm{O}$ estudo das redes nesse caso é útil para entender os padrões de relações, já que demonstra que os ministérios de agricultura dos países membros dependem mais diretamente das associações de produtores para coletar informações e para angariar apoio público, e que os agentes políticos regionais buscam primeiro alinhar-se com suas contrapartes políticas. Mais importante, propõe um método que permite a comparação entre países ou regiões, elemento que ainda é um limitador importante para o estudo de grupos de interesse.

Outra forma de estudar os padrões de relações de grupos de interesse entre si e com instâncias decisórias é a análise do relacionamento entre grupos de interesse e cortes supremas. Uma das principais referências para esse trabalho, dada a semelhança de métodos, é Box-Steffensmeier e Christenson (2014), que analisaram os padrões de participação de grupos de interesse em processos na suprema corte dos EUA na forma de amicus curiae. Os autores coletaram a informação da participação e de parceria de 5.291 grupos de interesse que entregaram 3.807 memoriais a juízes sobre 718 casos julgados entre 1930 e 2009. Os resultados apontam o aumento da consistente da participação de grupos de interesse na suprema corte dos EUA, com cada vez maior conectividade (graus Freeman), ao mesmo tempo em que a densidade, a transitividade e a centralização se mantiveram consistentemente baixa, alta e baixa, respectivamente, durante todo esse período. Além disso, lançou luz sobre os padrões de construções de coalizões.

Outra perspectiva ainda é trazida por Heaney (2014), que investiga a relação entre a reputação de influência de um determinado grupo e sua posição na rede de grupos de interesse. A hipótese é que grupos de interesse envolvidos em redes multiplex (definidas por redes de comunicação, participação em coalizões e coincidência de temas de políticas públicas) tendem a ter uma percepção mais precisa sobre a influência real dos grupos de interesse com os quais se relaciona, porque eles possuem mais informações sobre suas capacidades e modo de operação. Assim, em redes multiplex a reputação de influência poderia ser uma proxy confiável para a influência efetiva. O teste é feito sobre 168 entrevistas feitas com grupos de interesse na área de saúde pública nos EUA. Os resultados indicam que grupos de interesse tendem mais frequentemente a indicar como influentes (1) outros grupos com características semelhantes (homofilia), tais como tipo organizacional, ligações partidárias e outros, e (2) outros grupos com os quais mantêm relações, seja por meio da rede de comunicação, de coalizões, seja de temas. Isso indica que não há uma competição entre as duas explicações, homofilia e a participação em outras redes. 
As redes criadas entre grupos de interesses e parlamentares também foram objeto dos estudos Koger e Victor (2009) e McClurg \& Philips (2011). Koger e Victor (2009) indicam que grupos de interesse tendem a fazer doações para parlamentares que votam juntos, participem do mesmo comitê, sejam do mesmo partido, representem o mesmo estado ou enfrentem eleições difíceis, confirmando em grande medida a hipótese de homofilia para a formação de redes. McClurg e Philips (2011) buscam outra perspectiva e analisam as relações entre grupos de interesse e parlamentares por meio de doações da perspectiva de promoção de competição ou cooperação entre partidos. A adoção de estratégias de promoção de competição ou de cooperação entre partidos, segundo os autores, depende da estratégia do grupo de interesse, do nível de oposição ao status quo da política e de sua habilidade de articular apoio com parlamentares.

Outro estudo sobre redes de grupos de interesse, mais similar ao modelo aqui utilizado, é Lapira, Thomas e Baumgartner (2009), que mapearam grupos de interesse que atuaram em Washington por um período de dez anos a partir dos relatórios de atividades de grupos de interesses, que são mandatórios naquele país. Seus objetivos são mensurar o padrão das conexões entre lobistas e analisar a estabilidade desses relacionamentos ao longo do tempo. Na estrutura mapeada fica claro que alguns temas de políticas públicas são mais centrais e outros mais periféricos. Nos temas centrais a predominância é de lobistas contratados e especializados e, nos demais, a característica mais marcante passa a ser a utilização de pessoal interno e generalista. Ademais, o estudo aponta que a rede de relacionamentos de lobistas é estável ao longo do tempo, ainda que possa ser perturbada por mudanças externas. Tal como no Brasil, observa-se uma importante concentração da participação de grupos e interesse sobre um pequeno número de temas, mas os temas de maior incidência são diferentes do Brasil. Os temas com maior incidência de ação de grupos de interesse são, na ordem: 1) orçamento público, (2) saúde, (3) tributação, (4) energia e energia nuclear, (5) defesa, (6) comércio internacional, (7) Medicare e Medicaid, (8) transporte, (9) educação e (10) segurança nacional.

No entanto, apesar das aplicações acima, ainda há limites na aplicação do ferramental de análise de redes aos grupos de interesse. Os limites da aplicação da análise de redes a grupos de interesse estão, tal como aponta Ward, Stovel e Sacks (2011), (1) na ainda limitada relação entre os conceitos da Ciência Política e de redes e (2) em limites do método, tal como análises de redes de grande escala, dados dinâmicos, relações episódicas e redes. Especialmente no que tange a redes de grande escala e de relações não binárias, essas limitações de método são um limitador da capacidade de gerar análises a partir dos dados coletados.

\section{O desenho da pesquisa}

Os dados para a construção da rede de grupos de interesse foram coletados a partir das páginas da Câmara dos Deputados e do Senado Nacional. As comissões parlamentares publicam relatórios de audiências públicas e seminários com data, horário, título da reunião, participantes, seus cargos e instituições. A pesquisa buscou nessas fontes registrar todas as participações nos anos de 2011 e 2012, período a partir do qual se encontrou melhor qualidade nos relatórios.

A rede foi desenhada de forma que cada grupo de interesse seja um nó e as ligações entre eles foi estabelecida pela ação em uma mesma política pública. Para isso, de cada audiência pública e evento foi registrado seus participantes e a política pública em discussão. Todos os grupos de interesse que buscaram influência na mesma política pública foram ligados entre si. 
A rede resultante é, portanto, de uma rede completa, com foco multitemático e de todas as comissões do Congresso Nacional, permanentes ou não.

Grupos de interesse são definidos como qualquer associação, formal ou não, que busca influenciar políticas públicas. Grupos de interesses estatais foram retirados dessa pesquisa já que uma das perguntas do projeto mais amplo no qual este artigo se insere era a capacidade de a sociedade civil se fazer ouvir pelo aparato estatal. Mas a realidade é que atores estatais fazem parte do processo de influência e isso fica bastante claro, por exemplo, com as assessorias parlamentares dos ministérios, agências, autarquias e empresas estatais. Eles formam por si uma rede que age sobre outros poderes, mas também dentro de cada um deles. Contudo, para registrar esse fenômeno existe uma dificuldade metodológica ainda não superada neste trabalho. Diferenciar entre as ações de defesa de interesses e seu papel formal de representação de um poder perante o outro está longe de ser simples. Ou seja, ainda que exista um argumento razoável para incluir agentes públicos, a inclusão dependeria uma análise de cada participação, se ela se encontra dentro de suas atribuições formais ou não. Uma forma de remediar esse a dificuldade de separar funções foi a inclusão dos grupos de interesses de funcionários públicos, estes, sim, da sociedade civil, mas representantes de interesses de organizações públicas e seus funcionários, sem as restrições formais de competência típicas de um órgão público.

Outra restrição foi a exclusão de especialistas "isentos", muitas vezes professores universitários ou "especialistas". Apesar de parecem prima facie como isentos, sua participação frequentemente deve-se ao seu alinhamento com a visão de um grupo ou interesse específico. Esse tipo de representação impede o registro, de maneira estruturada, do grupo de interesse envolvido na ação.

Para garantir a comparabilidade com outros estudos (Lowery et al., 2012), os grupos de interesse foram categorizados segundo Schlozman (2012) e as políticas públicas segundo Baumgartner e Jones (2013). Schlozman (2012) procurou fazer um retrato mais detalhado dos tipos de grupos de interesse. A estrutura resultante consiste em 24 categorias com várias subdivisões. A segunda classificação necessária foi de políticas públicas, quando foi utilizado a classificação do policy agendas (Baumgartner \& Jones 2013), que registra informações sobre política públicas nos EUA desde a II Guerra Mundial. As duas estruturas mostraram-se bastante aderentes ao sistema político brasileiro, embora algumas categorias tivessem que ser adaptadas para o ambiente político brasileiro $^{2}$.

${ }^{2}$ Por exemplo, a categoria native americans, foi adaptada para indígenas e comunidades tradicionais.
Ainda, para permitir a comparações com populações de grupos de interesses de outros países, uma das opções deste trabalho é apresentar os coeficientes normalizados. Todas as análises estatísticas foram feitas pelo sistema UCINET6 e as principais referências técnicas em análise de redes foram Hanneman e Riddle (2005) e Kolaczyk (2010).

\section{Resultados}

Seguindo a estratégia comum na análise de redes, os resultados serão apresentados em duas partes. Na primeira serão analisadas as propriedades da rede como uma entidade própria. Na segunda o objetivo será de estudar os papéis e a importância de cada um dos nós. Nas duas, os resultados são acompanhados de discussões sobre as implicações dessas características para o ambiente político de grupos de interesse.

A primeira característica relevante da rede de grupos de interesse no Congresso Nacional é seu tamanho. A rede é grande e formada por 975 grupos de interesse. Por outro lado, existem na rede 81.668 ligações entre os grupos de 
interesse. A rede é altamente conectada, já que $93 \%$ dos grupos de interesse são conectados entre si de alguma forma.

Essas características permitem caracterizar um componente gigante, ou seja, um grande agrupamento que engloba quase a totalidade de grupos de interesses. Essas redes, tal como demonstra Janson et al. (1993) em experimentos aleatórios, surgem em geral quando uma rede possui mais de $1 / 2 \mathrm{n}-$ sendo $\mathrm{n}$ o número de nós $(\mu=3,93)$. Nesse caso, caracteriza-se a rede como sendo de grande robustez, com redundâncias e baixa capacidade de alguns nós restringirem o acesso de um subconjunto ao resto da rede. Em outros termos, uma estrutura que dificilmente seria destruída em caso da remoção de um de seus nós.

Essa robustez também pode ser confirmada com a análise estrutural, que investiga a ausência de "pontos fracos", "vulnerabilidades" ou "vazios". Uma busca por aqueles grupos de interesse que servem de conexão única entre um bloco nós e os demais nós da rede (bi-components), ou seja, grupos que funcionam como "pontos de corte", indicou apenas 22 blocos. Os blocos eventualmente formados são caracterizados por organizações de foco bastante específico e têm pouco significado para a rede como um todo.

Em termos políticos, significa que, caso algum grupo deixasse de atuar democraticamente, ele teria baixa capacidade de negar o acesso e arrastar demais grupos para fora do sistema democrático de representação junto ao parlamento. Quando se trata de uma democracia recente e ainda em consolidação, tal como a brasileira, essa característica tem grande relevância. Ela aponta que a sociedade civil participa do processo decisório. E mais, grupos interesses que em outros momentos da história brasileira foram relevantes em arrastar o país para longe da democracia hoje não teriam essa mesma capacidade.

Outra característica importante da rede é que existem poucas ligações entre os nós comparado ao que seria possível. Em um índice que parte de 0 (não há ligações) e cresce até 1 (situação em que todos os nós são ligados diretamente a todos os demais nós), a rede de grupos de interesse tem uma densidade (média de intensidade dos links) de apenas 0,086. No entanto, a distância média entre nós é pequena, de apenas 2,552. De modo mais simples, a média dos menores caminhos entre todos os nós é de apenas 2,552 e o desvio padrão é de 0,644. A maior distância entre grupos de interesse - o diâmetro da rede - é de 6.

Ainda, vale destacar a enorme incidência de clusters na rede. A análise de clusters busca identificar subconjuntos de grupos de interesse que possuem alta incidência de ligações entre si e tendem a se tornar mais próximos na análise espacial. Aqui o cálculo é sobre a densidade da vizinhança de cada um dos grupos de interesse, quando ele mesmo é retirado. Os resultados da rede de grupos de interesse indicam uma altíssima clusterização (vizinhanças bastante densas) por meio de um índice de clusterização ponderado de 1,969. A existência de clusters também podem ser vista nas Figuras 1 e 2, onde estão destacadas por cores cada um dos $36 \mathrm{k}$-cores identificados na rede.

Assim, a rede reúne, ao mesmo tempo, três características importantes: a baixa densidade da rede como um todo, a marcante presença de clusters e a distância curta entre seus nós. Essa conjugação permite a aproximação de outro modelo analítico de redes, o de small worlds. Neste modelo, a rede assume uma posição intermediária entre as redes regulares e as completamente aleatórias e são observadas na realidade em diferentes contextos, como a internet, biologia, produção científica e etc. Um efeito relevante dessas redes é que a transmissão do que quer que trafegue, nesse caso informação e influência, é mais rápida e mais ampla (Watts \& Strogatz 1998). 
Figura 1 - Mapa geral de grupos de interesse

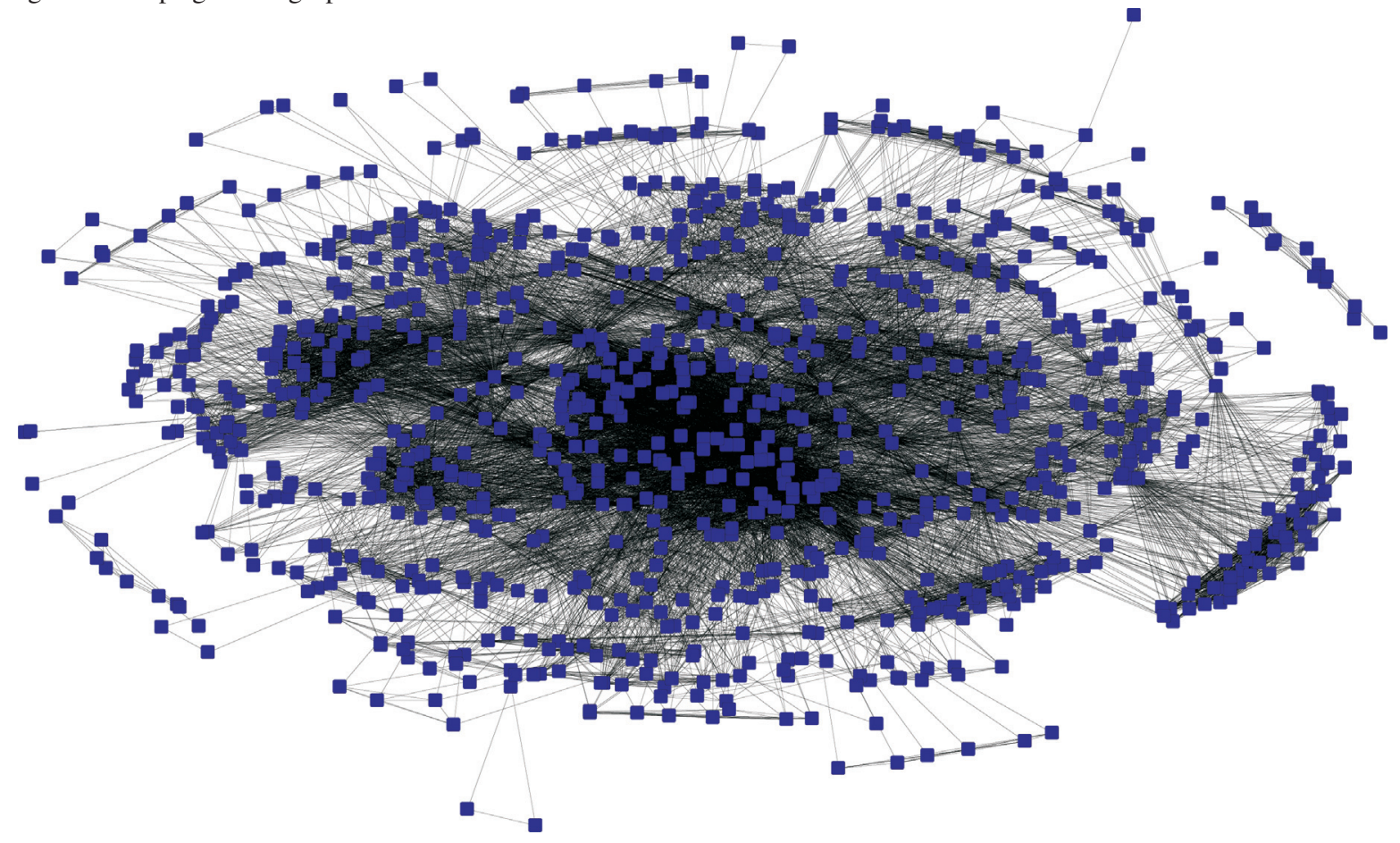

Fonte: o autor.

Figura 2 - Mapa geral de grupos de interesse com k-cores destacados

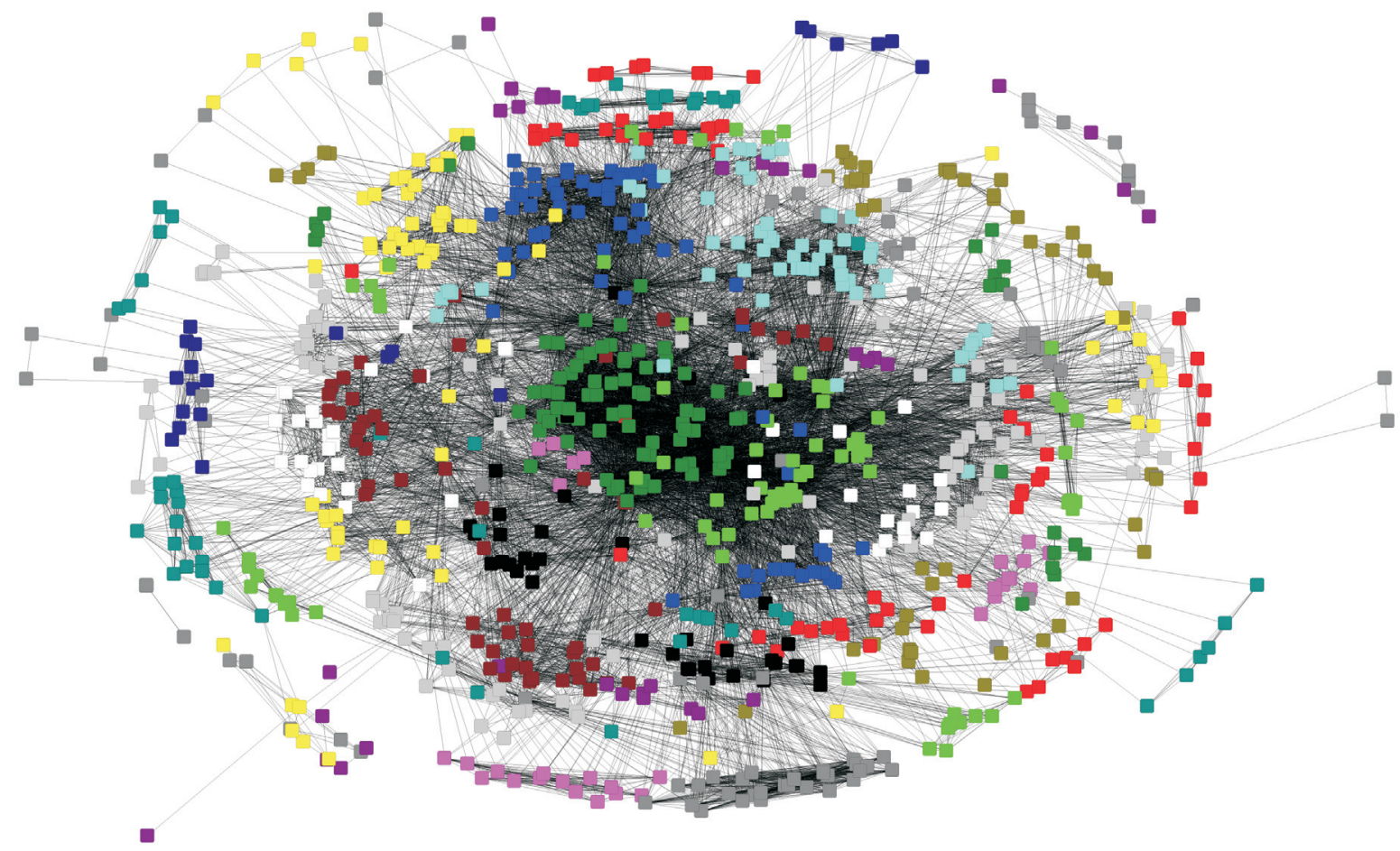

Fonte: o autor.

Mais uma vez, redes small world podem ter efeitos relevantes do ponto de vista político. Em primeiro lugar, esse é um jogo entre conhecidos ou entre conhecidos de conhecidos, já que as distâncias são curtas. Espera-se com isso 
${ }^{3}$ O Índice E-I varia de 1, quando todas as ligações são feitas entre grupos de interesse de diferentes categorias, até -1 , quando as ligações são todas internas. também um fluxo de informações rápido entre grupos de interesse e a articulação de coalizões (Figuras 1 e 2).

Segundo, em decorrência da elevadíssima clusterização, observa-se um ambiente político com alto nível de especialização e pequeno espaço para negociações entre grupos de interesse que atuem em matérias distintas, como barganhas cruzadas.

Outra análise importante é a da perspectiva dos diversos tipos de grupos de interesse, segundo a classificação de Schlozman (2012). Nesse caso, os resultados assumem nuances sem serem alterados substantivamente. Em primeiro lugar, tal como pode ser visto na Tabela 1, grupos voltados para o interesse público possuem a densidade média mais baixa, 0,076 , provavelmente resultante da grande diversidade de temas que estão incluídos nesta categoria. Da mesma forma, chama atenção a coesão de sindicatos e grupos de direitos civis, raciais, de minorias e nacionalidades, que, apesar de contarem com número relativamente grande de grupos de interesse, terminam por ser mais conectados. Isso indica uma capacidade superior de sindicatos e grupos de direitos civis, raciais e nacionalidades de construírem coalizões entre si e um padrão de ação conjunta. De um modo geral, redes maiores tendem a ser menos densas e esses dois agrupamentos fogem dessa regra.

A densidade superior das subredes de sindicatos e de grupos de direitos civis, raciais, de minorias e nacionalidades, pode ser interpretada como reflexo e consequência de maior capital social acumulado por esses grupos. $\mathrm{O}$ acúmulo desse capital social é materializado por meio da existência de instituições de articulação e ferramentas para diminuir os custos de intercâmbio de informações e construção de coalizões, que merecem uma investigação própria.

Essa (in)capacidade de mobilizar redes para ação concertada e a redução (o aumento) dos custos de transmissão de informações deve se reverter em maior (menor) influência. No caso de sindicatos e de grupos de direitos civis e raciais, a capacidade de articular e mobilizar deve resultar em maior influência desses grupos em suas políticas setoriais.

Outro ângulo de análise relevante é o padrão de relações de grupos de interesse interno e externo a sua categoria, também conhecido como Índice E-I. De uma forma geral, predominam na rede as relações entre grupos de interesse de categoria distinta, com um índice E-I de $0,388^{3}$. Isso significa que em boa parte das vezes os grupos participam em debates com outros que possuem de natureza distinta, o que leva, muitas vezes, ao enfrentamento entre pontos de vista distintos, tal como é razoável imaginar do processo democrático.

Tabela 1 - Tipos de Grupos de Interesse e Densidade Interna - para categorias com pelo menos 20 grupos de interesse

\begin{tabular}{lccc}
\hline Tipo de Organização & Número de organizações & Número de ligações & Densidade \\
\hline D - Sindicatos & 129 & 12502 & 0,757 \\
N - Grupos de direitos civis, raciais, de minorias e & 47 & 1634 & 0,756 \\
nacionalidades & & & 0,17 \\
R - Grupos de deficientes ou portadores de doenças & 35 & 202 & 0,161 \\
E - Agricultura & 37 & 2074 & 0,134 \\
C - Associações profissionais & 125 & 48 & 0,126 \\
G - Saúde & 20 & 12730 & 0,124 \\
B - Negócios & 321 & 1626 & 0,076 \\
K - Interesse Público & 147 & & \\
\hline
\end{tabular}

Fonte: o autor. 
Tabela 2 - Relacionamentos de grupos de interesse internos e externos a sua categoria - para categorias com pelo menos 20 grupos de interesse

\begin{tabular}{|c|c|c|c|c|}
\hline & Ligações internas & Ligações externas & Total & E-I \\
\hline G - Saúde & 42 & 412 & 454 & 0,815 \\
\hline E - Agricultura & 128 & 799 & 927 & 0,724 \\
\hline K - Interesse Público & 824 & 3125 & 3949 & 0,583 \\
\hline C - Associações profissionais & 950 & 3386 & 4336 & 0,562 \\
\hline D - Sindicatos & 2168 & 4393 & 6561 & 0,339 \\
\hline $\mathrm{R}$ - Grupos de deficientes ou portadores de doenças & 198 & 390 & 588 & 0,327 \\
\hline $\begin{array}{l}\mathrm{N} \text { - Grupos de direitos civis, raciais, de minorias e } \\
\text { nacionalidades }\end{array}$ & 678 & 1323 & 2001 & 0,322 \\
\hline B - Negócios & 4538 & 5391 & 9929 & 0,086 \\
\hline
\end{tabular}

Fonte: o autor.

${ }^{4}$ Isso revelaria também uma menor capacidade de articulação e influência e uma pulverização de interesses.
Chama atenção na Tabela 2, todavia, que os grupos de negócios relacionam-se menos frequentemente com grupos pertencentes a outras categorias. Duas razões podem explicar essa diferença de comportamento isoladamente ou em conjunto: 1) uma escolha por estabelecer diálogos apenas com grupos com posicionamentos mais convergentes, o que poderia chamado de uma estratégia de "pregar para convertidos" ou (2) a existência de conflitos internos em temas com baixo interesse para as demais categorias ${ }^{4}$.

Por outro lado, grupos de interesse da área de Saúde, Agricultura, Interesse Público e Associações Profissionais participam principalmente de debates em que há predominância de grupos de tipos diferentes de seus próprios. Isso indica que estabelecem negociações entre pontos de vista diferentes e a busca de criar coalizões mais heterogêneas e provavelmente mais fortes.

Assim, de uma forma geral, a rede de grupos de interesse pode ser caracterizada como robusta, de baixa densidade, marcada por uma alta especialização em torno de políticas públicas específicas. Mesmo assim, trata-se de um jogo entre conhecidos ou de conhecidos de conhecidos, já que a distância média entre grupos é de 2,5 e a distância máxima é 6. Para grupos de interesse público, empresariais, associações profissionais, agricultura e grupos de deficientes ou portadores de doenças, a baixa articulação interna e o elevado número de participantes devem aumentar a dificuldade de encontrar posições comuns (dada a diversidade de pontos de vista e até mesmo o conflito interno) e restringir sua capacidade de influência. O contrário deve ser observado para os grupos militantes na área de sindicatos e grupos de direitos civis, raciais, minorias e nacionalidades. A análise de relações internas e externas das categorias demonstra que o padrão é a relação entre "diferentes", o que está em linha com a expectativa de que os debates sejam feitos para construir consensos entre pontos de vistas distintos. O destaque negativo, nesse caso, está para os grupos de negócios, que apresentam um padrão de diálogo entre "iguais", seja porque buscam evitar o conflito "externo", seja porque o nível de conflito "interno" é elevado. Finalmente, a rede é robusta, na medida em que mesmo com a retirada de nós (por exemplo com a saída do processo democrático, o que significa sua morte) tende a não isolar outros grupos, já que existem redundâncias. Para a democracia, a implicação de uma rede robusta é que ela é mais difícil de ser destruída, mesmo que vários de seus atores abandonem o processo democrático, por haver ligações redundantes.

No nível macro, o nível de centralização da rede, ou seja, o grau em que se pode dizer existe desigualdade entre os nós (grupos de interesse) é baixo, de 
5 Para a listagem desses grupos foi escolhido o procedimento de núcleo categórico do UCI 6 for Windows e apresentou resultados consistentes em vários cenários de teste. $\mathrm{O}$ algoritmo utilizado foi o CORR. Detalhes sobre o procedimento podem ser encontrados em Borgatti e Everet (1999). apenas 0,305 . Uma rede em formato de estrela tem a mais alta centralização, com índice 1. Por outro lado, uma rede em círculo tem a centralização mais baixa, com 0 .

A rede de grupos de interesse em estudo apresenta a característica oposta, onde fica patente a não existência de um grupo de interesse (ou de um subconjunto) capaz de exercer de maneira não contestada a influência. Isto implica que nenhum dos de interesse grupos (ou subconjuntos destes) pode reclamar para si um papel de hegemonia e que este ambiente, ao fim, é marcado por uma alta competição e baixas barreiras de entrada.

Mesmo assim, o perfil de participação de grupos de interesse demonstra alta variação. Em média, os grupos de interesse têm 83,96 graus Freeman (ligações), mas o desvio padrão é de 197 graus e o número efetivo vai de 0 graus até 2.826 . O Gráfico 1 representa essa distribuição e aponta a existência de uma rede bastante heterogênea com uma distribuição de natureza exponencial.

Desse modo, uma indagação relevante é se haveria nessa rede algum conjunto de grupos de interesse que teria uma capacidade muito maior de mobilização e articulação ou poderiam, por outro lado, servir como hubs de informação e articulação? Em outros termos, haveria nessa rede um núcleo de grupos de interesse?

O núcleo de uma rede é um subconjunto de nós (grupos de interesse) que possui relações mais densas entre si e com a periferia do que esta consigo própria. A existência de um núcleo pode ser comprovada, portanto, pela análise das densidades das relações.

Tabela 3 - Densidade de relação do núcleo e da periferia

\begin{tabular}{lcc}
\hline & Núcleo & Periferia \\
\hline Núcleo & 57,015 & 0,876 \\
Periferia & 0,876 & 0,058 \\
Starting fitness: 0,717 & & \\
Final fitness: 0,717 & & \\
\hline
\end{tabular}

Fonte: o autor.

Gráfico 1 - Graus Freeman de influência não normalizados

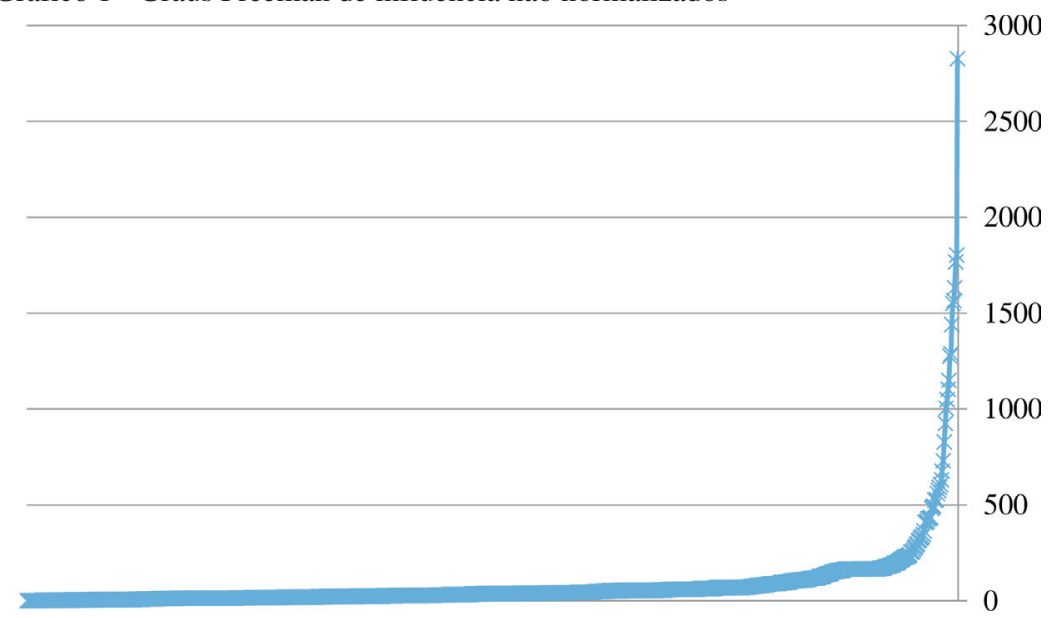

Fonte: o autor. 
${ }^{6}$ É importante ressaltar que se trata da influência sistêmica. Esses grupos podem exercer papeis centrais em determinadas políticas públicas, mas esse diagnóstico está fora do escopo desse estudo.
No caso da rede de grupos de interesse no Poder Legislativo entre 2011 e 2012, a resposta é sim; existe claramente um núcleo de organizações com um papel de coordenação sistêmica, tal como se pode ver na Tabela $3^{5}$.

O núcleo é composto por 14 grupos de interesse (1,43\% da amostra) e possui um perfil relevante para os interesses dessa pesquisa, conforme pode ser notado na Tabela 4. Metade dos integrantes do núcleo da rede é composta de centrais sindicais (CTB, CUT, CONLUTAS, NCST e UGT) e de suas organizações afiliadas (DIEESE e FST). Ainda na pauta dos trabalhadores se destacam os sindicatos e associações de funcionários públicos (SINAIT - auditores do trabalho, ANPT - procuradores do trabalho, ANAMATRA - juízes do trabalho, ANFIP - auditores fiscais) e uma associação de aposentados (COBAP), que age em estreita parceria com as centrais sindicais. Entre eles, apenas dois grupos são empresariais, a CNI e a CNC.

Observa-se, portanto, uma participação muito superior de grupos de interesses trabalhistas ao dos grupos empresariais a e inexistência dos demais tipos de grupos no núcleo da rede. Vale ressaltar que são essas as organizações com maior capacidade de mobilização e de articulação. Também estão em posição estruturalmente privilegiada. Esse privilégio consiste em maior capacidade de coletar e repassar informações, mobilizar outras organizações e canalizar recursos de conhecimento em políticas públicas ou financeiros.

As Figuras 3 e 4, a seguir, representam a existência de um núcleo. Os nós maiores ficam concentrados no núcleo enquanto a periferia da rede mantém os nós com menor influência sistêmica ${ }^{6}$, tal como medido pela proximidade. Observa-se também a participação de centrais sindicais, de associações empresariais e de sindicatos de servidores públicos.

Outra forma de investigar a influência/centralidade de grupos de interesse é a análise a partir dos tipos de grupos de interesse. A discussão teórica é relevante já que boa parte da discussão sobre grupos de interesse gira ao redor da questão: grupos de negócios são caracterizados por elevada assimetria de influência e podem exercer a hegemonia? A resposta empírica a essa questão tem que buscar diferenças relevantes de centralidade entre os tipos de grupos de

Tabela 4 - Integrantes do núcleo da rede de grupos de interesse

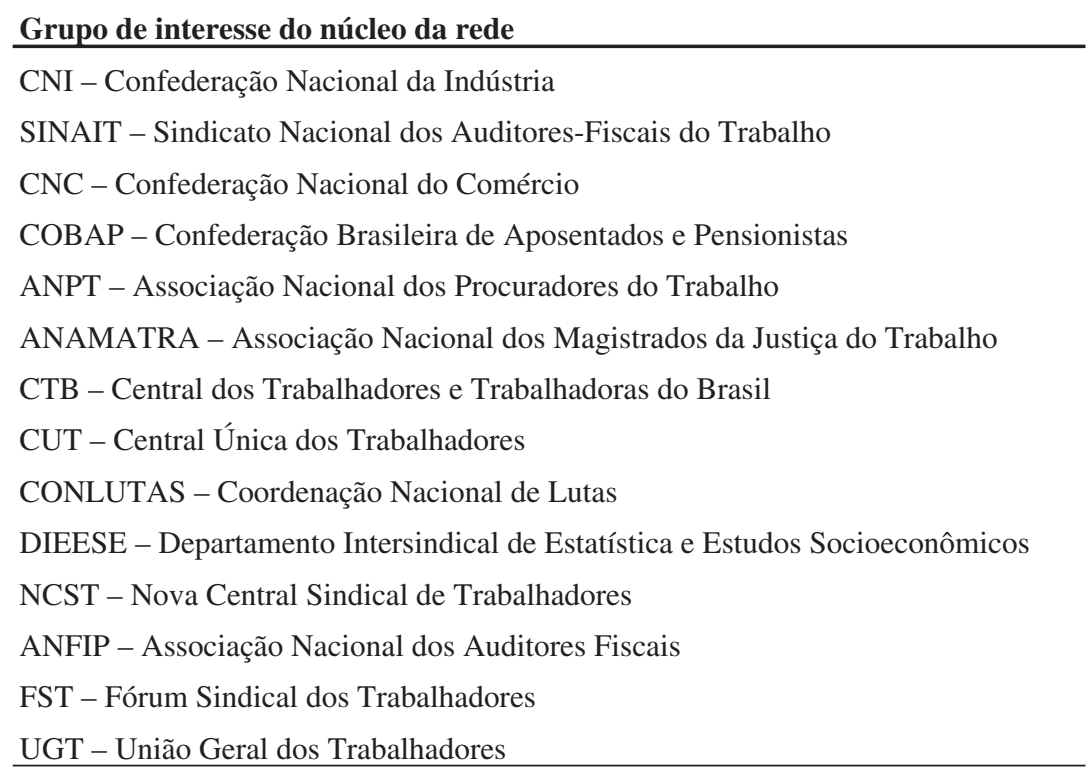

Fonte: o autor. 
Figura 3 - Mapa geral da rede com nós coloridos por tipo de grupo de interesse e com tamanho de acordo com graus Freeman

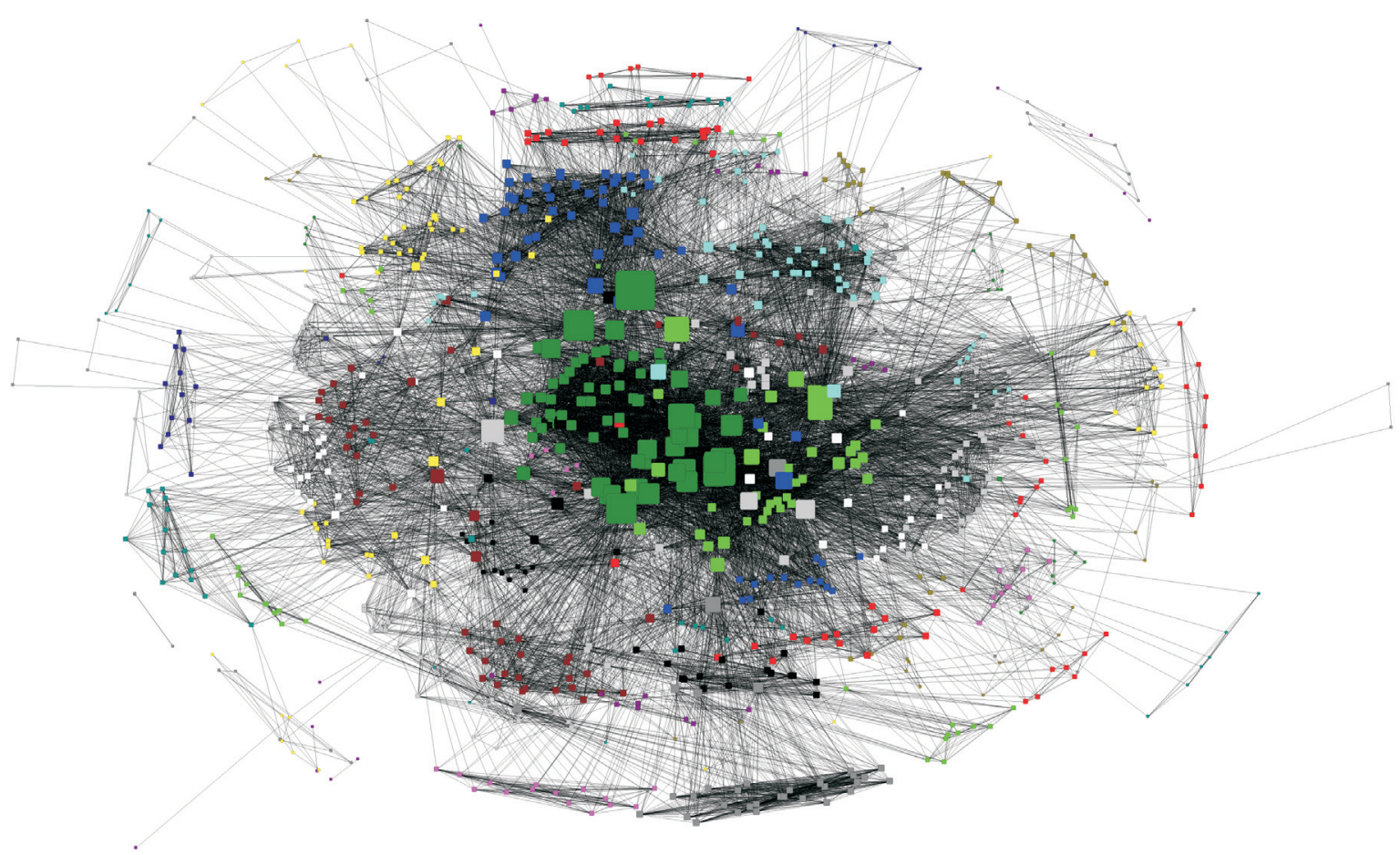

Fonte: o autor.

Figura 4 - Mapa do núcleo da rede

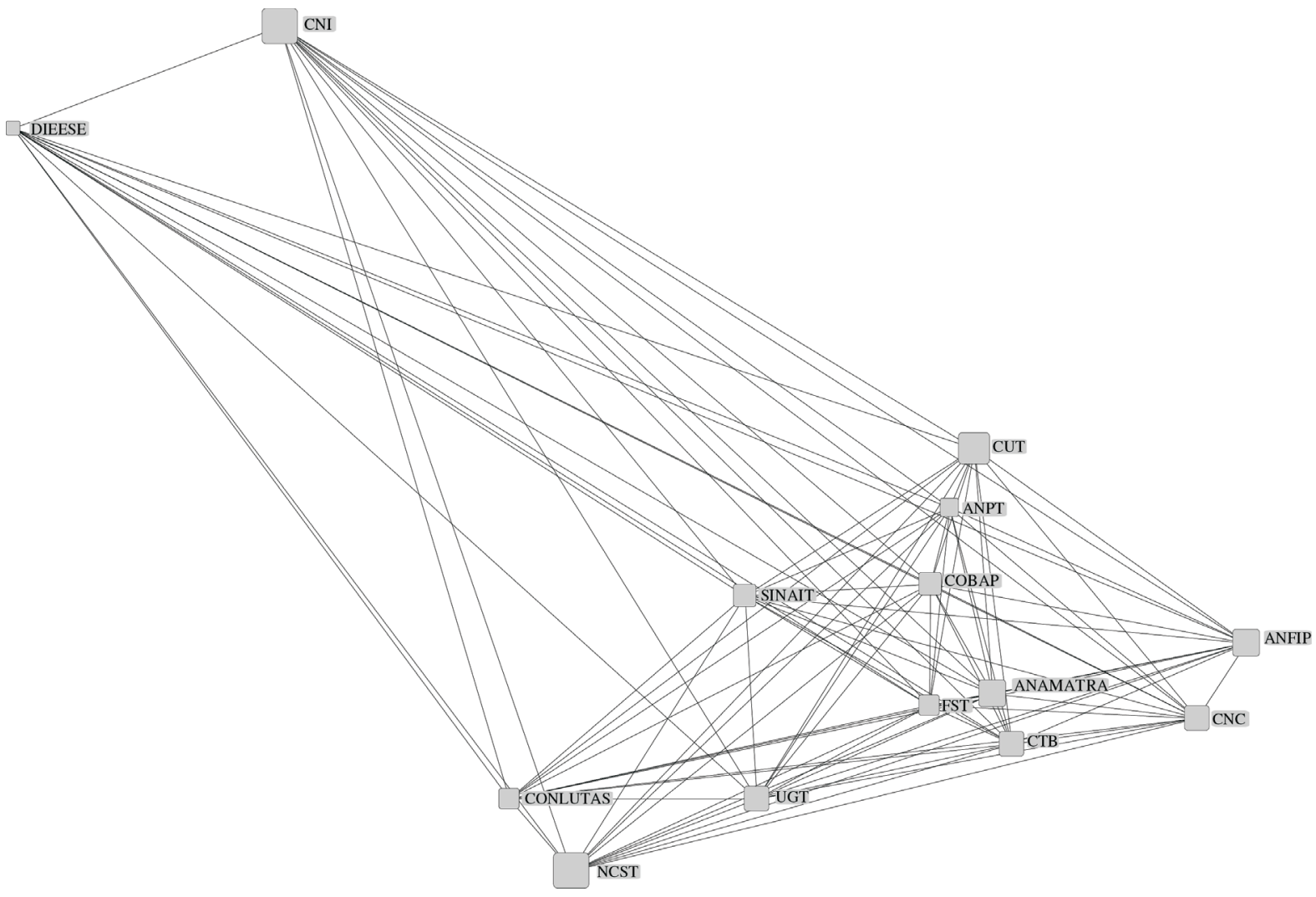

Fonte: o autor. 
${ }^{7}$ Dentro de tipos de grupos de interesse existe heterogeneidade de interesses e é importante lembrar que muitas vezes se observam conflitos internos. De modo que seria um salto empiricamente não sustentado afirmar que os 4 tipos mais influentes sejam capazes de dominar o ambiente político. interesse. A técnica utilizada aqui é reduzir cada um dos tipos de grupos de interesse a apenas um nó, que consolida as ligações de todos os demais grupos de interesse do mesmo tipo.

O resultado da Tabela 5 demonstra que existem quatro tipos de grupos de interesse com maior influência: negócios, associações profissionais, grupos de interesse público e sindicatos. Os demais também apresentam indicadores razoáveis quando consideradas duas ligações (ou graus), o que resulta da alta conectividade da rede. Portanto, assimetrias de influência são observadas quando os tipos de grupos de interesse são o objeto da análise, mas, mais uma vez, não é possível identificar um tipo de grupo de interesse que possa ser caracterizado como hegemônico ${ }^{7}$.

Outra perspectiva na análise de redes é a mensuração da importância de cada um dos nós a partir das relações estabelecidas (ou fluxos) e dos papeis que cada nó desempenha a partir do conceito de centralidade. As aplicações da teoria das redes para nas Ciências Sociais têm utilizado a medida de importância de cada um dos nós como uma proxy da influência ou de poder de cada agente social, tal como mostram Degenne e Forsé (1999, cap. 6 e 7).

Quando o objeto do estudo são os grupos de interesse e suas relações de cooperação e oposição em políticas públicas, o conceito de influência "em rede" assume uma importância ainda maior. Além de permitir medidas objetivas feitas a partir de uma metodologia consolidada, a influência, medida pela centralidade, passa a ser considerada como resultado das interações estabelecidas entre grupos de interesse, a rede. Abre-se então uma perspectiva mais ampla em que não apenas de características intrínsecas ou extrínsecas de grupos

Tabela 5 - Graus de influência de tipos de grupos de influência

\begin{tabular}{|c|c|c|}
\hline & \multicolumn{2}{|c|}{ Graus normalizados } \\
\hline & 1 degrau & 2 degraus \\
\hline B - Negócios & 0,873 & 0,96 \\
\hline C - Associações profissionais & 0,815 & 0,968 \\
\hline K - Interesse Público & 0,783 & 0,975 \\
\hline D - Sindicatos & 0,702 & 0,962 \\
\hline $\mathrm{N}$ - Grupos de direitos civis, raciais, de minorias e nacionalidades & 0,349 & 0,944 \\
\hline S - Serviço social e pobreza & 0,3 & 0,949 \\
\hline $\mathrm{J}$ - Estrangeiros & 0,29 & 0,933 \\
\hline W - Centros de Conhecimento (think tanks) e outras organizações de pesquisa sem fins lucrativos & 0,284 & 0,959 \\
\hline H - Governo & 0,274 & 0,925 \\
\hline O - Idade & 0,232 & 0,915 \\
\hline E - Agricultura & 0,229 & 0,843 \\
\hline G - Saúde & 0,21 & 0,906 \\
\hline $\mathrm{R}$ - Grupos de deficientes ou portadores de doenças & 0,195 & 0,906 \\
\hline F - Educação & 0,17 & 0,886 \\
\hline U - Artes e Cultural & 0,168 & 0,876 \\
\hline X - Outros & 0,096 & 0,767 \\
\hline Q - Orientação sexual & 0,06 & 0,794 \\
\hline L - Eleitoral ou partidário & 0,046 & 0,585 \\
\hline P - Gênero & 0,042 & 0,677 \\
\hline
\end{tabular}

Fonte: o autor. 
8 Outro modo de medir a influência de grupos de interesse utilizada na literatura é sua capacidade de alcançar os resultados esperados. Essa perspectiva, todavia, não é a completa nem definitiva. Muitas vezes grupos de pressão atuam para sensibilizar (sem a pretensão de alcançar um resultado objetivo) interlocutores ou apenas para bloquear ações de grupos opositores. Por isso, a visão de alcançar apenas resultados é limitadora na medida em que desconsidera uma das características fundamentais que o jogo de pressões e contrapressões típicas da relação entre a sociedade civil e a o estado. de interesse são determinantes de sua influência, tal como tem sido o foco das pesquisas até o momento. $\mathrm{O}$ "poder" então passa a ser um conceito relacional, baseado na capacidade de fazer parte da rede e no modo de participação do debate político. Esse posicionamento naturalmente também está ligado aos recursos disponíveis ou suas estratégias, mas ela se consolida no espaço conseguido para participar do debate ${ }^{8}$.

Mesmo sendo aceita como uma proxy para a influência, ainda existem diferentes formas de medir a centralidade de uma rede baseada no acesso de grupos de interesse ao parlamento brasileiro. Existem três grandes linhas para mensurar em termos genéricos a centralidade. A primeira está baseada nas ligações que cada nó (graus, degrees) possui direta ou indiretamente. Na base dessa medida estão as noções de capacidade de arregimentar recursos da rede e na redução dos custos de transação. Quanto maior o número de ligações de um grupo de interesse pode-se dizer que ele possui mais prestígio e proeminência, sendo assim mais influentes.

A segunda forma de medir influência é a proximidade (closeness) de um grupo de interesse de todos os demais. Em outros termos, pela sua capacidade de alcançar todos os demais nós, ou grupos de interesse, com o menor número de intermediários. Nesse caso, um grupo de interesse com alta proximidade é em geral tido como uma referência a partir do qual os demais se medem e possui, ainda, uma maior capacidade de se fazer ouvido.

A terceira maneira de mensurar a centralidade busca identificar posições de intermediário entre nós (betweeness). Ser considerado como um intermediário importante, ou broker, abre a possibilidade impor condições para que relações sejam feitas, que nós sejam isolados ou mesmo que certos contatos sejam evitados ou promovidos.

Foram abaixo, na Tabela 6 , mostrados apenas os dez primeiros nas três medidas. Os resultados indicam que apenas três organizações figuraram nas três medidas como importantes, por conta de seu prestígio e conexões, por exercerem um papel de referência e também por atuarem como intermediárias relevantes: NCST, CNA e CNI.

Nota-se, ademais, uma diversidade relevante que combina principalmente centrais sindicais de trabalhadores, organizações empresariais, sindicatos de funcionários públicos, órgãos de classe e organizações de defesa do consumidor.

Outra forma de analisar os papeis é estudar a posição de cada um dos grupos de interesse como broker, ou seja, como um intermediário importante. Mesmo não estando no núcleo da rede, grupos de interesse podem assumir papeis relevantes com estratégias diferenciadas de engajamento no Congresso Nacional. Esses papeis distintos também permitem representar e refletir diferentes perspectivas de um fenômeno complexo como a influência, sem simplificar demasiadamente esse conceito. Assim, permitem visualizar não apenas os agentes centrais, mas também certos grupos com papeis relevantes na circulação de informações, influência e recursos. Os papeis desenvolvidos por Gould e Fernandez (1989) estão descritos na Tabela 7, a seguir, e procuram refletir algumas dessas estratégias.

Na Tabela 8 reaparecem alguns dos grupos que figuraram no núcleo ou com índices de influência altos, mas também surgem novos grupos que possuem estratégias diferenciadas de inserção nas discussões. Acabam, assim, assumindo papeis importantes para a rede como um todo. Entre os coordenadores estão as associações setoriais de supermercados e de têxteis. No grupo de consultores, surgem outros, como o IDEC, de consumidores, a Contag, da agricultura familiar e o Sebrae, serviço de apoio a micro e pequenas empresas. Assim, a 
Tabela 6 - Ordem de influência a partir de quatro medidas de centralidade

\begin{tabular}{|c|c|c|c|}
\hline Organização & Graus (Degree Freeman) & Proximidade (Closeness) & Ligação (Betweeness) \\
\hline & Ligações diretas & $\begin{array}{l}\text { Facilidade de alcançar } \\
\text { demais nós da rede }\end{array}$ & $\begin{array}{l}\text { Ligação entre agentes mais } \\
\text { importantes para a rede }\end{array}$ \\
\hline Papel & Prestígio & Referência para o grupo & Intermediário \\
\hline NCST & 1 & 3 & 6 \\
\hline CNA & 2 & 2 & 1 \\
\hline CUT & 3 & 5 & 10 \\
\hline UGT & 4 & 10 & \\
\hline ANAMATRA & 5 & 7 & \\
\hline FST & 6 & & \\
\hline СТВ & 7 & & \\
\hline SINAIT & 8 & & \\
\hline $\mathrm{CNI}$ & 9 & 4 & 5 \\
\hline COBAP & 10 & & \\
\hline $\mathrm{CNM}$ & & & 3 \\
\hline CFM & & 6 & 4 \\
\hline $\mathrm{OAB}$ & & 1 & 2 \\
\hline \multicolumn{4}{|c|}{ Liderança Indígena } \\
\hline CONTAG & & 8 & 8 \\
\hline ANFIP & & 9 & \\
\hline IDEC & & & 7 \\
\hline Intervozes & & & 9 \\
\hline
\end{tabular}

Fonte: o autor.

Tabela 7 - Tipos de intermediário

\begin{tabular}{ll}
\hline Tipo & Descrição \\
\hline Coordenador & Ligação entre nós de mesmo tipo \\
Gatekeeper & Quando o nó de origem é de categoria distinta \\
Representante & Quando o nó de destinação é de categoria distinta \\
Consultor & O intermediário é parte de um grupo e os outros dois são de categoria distinta \\
Liason (facilitador) & Quando cada um grupos pertence a categorias diferentes \\
\hline
\end{tabular}

Fonte: o autor.

importância dos grupos de interesses mais centrais fica reforçada, mas também destaca-se a opção por estratégias específicas para potencializar a influência de grupos através de um posicionamento mais central na rede.

\section{Conclusões}

De volta às perguntas iniciais, pode-se afirmar que existe uma população de grupos de interesse bastante diversificada atuando no Congresso Nacional. A rede completa de grupos de interesse é de baixa densidade de uma forma geral, ou seja, há poucas ligações entre grupos de interesse. Outra característica relevante é a formação de clusters muito bem definidos ao redor de políticas públicas específicas, com muitas organizações especializadas em poucas polí- 
Tabela 8 - Dez primeiros grupos de interesse segundo tipo de intermediário

\begin{tabular}{lcccc}
\hline Coordenador & Gatekeeper & Representante & Consultor & Liason \\
\hline CNA & CNA & CNA & OAB & OAB \\
CNI & CNI & CNI & IDEC & CNA \\
NCST & NCST & NCST & NCST & CONTAG \\
CNC & OAB & OAB & CNM & CNM \\
ABRAS & CUT & CUT & CNA & NCST \\
CUT & CFM & CFM & CONTAG & CUT \\
OCB & CNC & CNC & ANAMATRA & CFM \\
UGT & UGT & UGT & CFM & CNI \\
CFM & ANAMATRA & ANAMATRA & SEBRAE & ANFIP \\
ABIT & ANFIP & ANFIP & ANFIP & COBAP \\
\hline
\end{tabular}

Fonte: o autor.

${ }^{9}$ Uma hipótese alternativa seria que esses grupos de negócios são altamente organizados a ponto de a representação ser feita quase que exclusivamente pela CNA, CNC e CNI, fazendo com que apenas dissidentes sejam representados individualmente. ticas públicas. Caracteriza-se, dessa forma, como uma rede altamente especializada. Essas características devem produzir efeitos como a (1) especialização de grupos de interesse, (2) pouco espaço para negociações cruzadas entre grupos de interesse e (3) dificuldades para a construção de coalizões amplas. A rede forma um componente gigante, já que quase todos os grupos de interesse podem ser alcançados através das ligações existentes.

Por outro lado, as distâncias entre grupos de interesse são pequenas, graças a presença de hubs, ou seja, nós com alta conectividade e que assumem a função de centrais de distribuição de informações e mobilização de recursos. A rede, assim, parece se aproximar dos modelos small worlds e componente gigante, que são conhecidas pela alta resiliência, estabilidade, facilidade de disseminação de informações e capacidade de mobilização.

O estudo dos relacionamentos dos tipos de grupos de interesse também possibilitou análises relevantes do ponto de vista teórico. As densidades de relacionamentos internos de tipos de grupos de interesse permitem explorar suas respectivas capacidades de articulação. Com isso, lançou luzes sobre a existência de instituições de articulação e sobre a acumulação de capital social desses segmentos. Destacam-se positivamente em sua capacidade de articulação interna os sindicatos e os grupos de direitos raciais, de minorias e nacionalidades.

O resultado sugere pesquisas adicionais para explicar os mecanismos de articulação interna e construção de capital social. Neste aspecto, ainda chama atenção também a baixa articulação interna de grupos de negócios ${ }^{9}$ e de grupos de interesse público.

As relações entre tipos de grupos de interesse permitem investigar o padrão de participação no debate democrático. O modelo pluralista propõe diversidade de interesses e grupos de interesses e um processo de interação entre esses. Seria razoável, nesse modelo, que boa parte das participações em audiências públicas fosse entre grupos de interesse de diferentes tipos. E, de fato, a interação com grupos de diferentes tipos é confirmada principalmente para grupos de interesse da área da saúde e agricultura. Por outro lado, os negócios parecem estar mais próximos do modelo de "pregar para convertidos".

Em termos gerais, uma conclusão relevante deste estudo é que as redes de grupos de interesse estão longe de ser marcadas por uma hierarquia rígida. A conexão dessa rede de baixa densidade e alta especialização é feita por grupos de interesse que funcionam como hubs ou centros de articulação. Do ponto de 
vista teórico, a existência desses hubs também aponta de que é necessário dar maior atenção a esses grupos de interesse que exercem esse papel. Isso porque eles assumem funções mais importantes para o funcionamento do sistema do que a defesa de seus próprios interesses. No fim, eles desempenham uma função pública de manutenção do sistema por meio da distribuição de informações e mobilização e recursos.

A influência de grupos de interesse e, mais especificamente, a assimetria de influência, foi o outro eixo norteador do trabalho. Nesse caso, a opção foi utilizar o acesso a processos decisórios como uma proxy para a influência de grupos de interesse. A primeira constatação é que existe um subconjunto de grupos de interesses que possui relações mais intensas entre si e com os demais que pode ser chamado de núcleo e pode, portanto, ser identificado como aqueles de maior influência. Mesmo no que se refere aos fluxos de informação, outro aspecto da influência, um subconjunto de grupos de interesse destacados bem semelhante surge. Outras formas de mensuração da centralidade foram testadas e resultados semelhantes puderam ser notados. Ainda assim, a rede tem baixa centralização.

Ainda, esses resultados demonstram que existem assimetrias de influência entre tipos de grupos de interesse, com a presença de um núcleo central de articulação e mobilização diversifica. Nenhum grupo de interesse ou tipo de grupo de interesse está sequer perto de exercer a hegemonia dada a baixa centralização da rede e até mesmo a variância dos graus de influência. Ao contrário, a análise mostra uma diversidade e uma disputa política relevantes entre grupos de interesse. Esses resultados servem, portanto, para rejeitar a hipótese de predominância de grupos de interesses de negócios, mas faz ressaltar a importância de grupos de interesses de funcionários públicos que tem sido em larga medida ignorada.

Naturalmente, esse estudo possui limites, derivados de suas escolhas. Em primeiro lugar, de acordo com as bases de dados e da escolha de método, essa pesquisa está baseada em uma fotografia. Ela retrata a rede de influência formal em um curto período de tempo (2011-2012) apenas sobre o poder Legislativo Federal. Essas relações são, no entanto, dinâmicas, têm uma característica informal e envolvem outros loci de influência, como os poderes Executivo e Judiciário.

Outra limitação importante que é o conceito de influência. Tal como o de poder, está sujeito a diferentes interpretações e, como consequência, sua mensuração é bastante complexa. Assim, a análise de redes e a centralidade de cada um dos atores será apenas uma medida imperfeita, mas, espera-se, ainda assim útil da influência de cada um dos grupos de interesse. A medida de influência, considerada essa restrição, pode servir para identificar os principais atores envolvidos no processo de influência e, com isso, uma das estruturas do processo político brasileiro. Mas não pode ser levada em como uma fórmula definitiva ou única para mensurar a influência.

Outro limite importante está relacionado com uma restrição da análise somente de grupos de interesse da sociedade civil. Naturalmente, há grupos de interesse de organizações públicas e que são bastante atuantes no Congresso, tal como aponta Santos (2014). Essa exclusão impõe um limite ao trabalho que tem razões apriorísticas e substantivas. Primeiro, esse estudo inseriu-se em uma pesquisa sobre o papel da sociedade civil na democratização brasileira dos últimos 20 anos. Segundo, é um desafio diferenciar as atribuições formais de relações entre os poderes e as ações de lobby dessas burocracias.

Como ainda estamos nos primeiros passos na investigação de grupos de interesse no Brasil, resta um longo caminho a ser percorrido. Assim, apenas 
uma lista limitada de próximos passos pode ser apresentada nesse momento. Em primeiro lugar, é necessário elaborar bases de dados que envolvam os poderes Executivo e Judiciário e ampliar os horizontes temporais. Ainda, seria importante evoluir para desenvolver testes estatísticos dos principais enunciados teóricos sobre grupos de interesse. Finalmente, é importante também evoluir para medidas alternativas de influência e para o estudo aprofundado de grupos de interesse, principalmente daqueles que possuem o papel de $h u b$.

Pablo Cesário (pscesario@yahoo.com.br) é Pós-Doutorando no Núcleo de Pesquisa em Políticas Públicas da Universidade de São Paulo (USP) e Doutor em Relações Internacionais pela Universidade de Brasília (UnB). Vínculo institucional: Núcleo de Pesquisa em Políticas Públicas, USP, São Paulo, SP, Brasil.

\section{Referências}

Baumgartner, F.R. \& Jones, B.D., 2013. Topics Codebook. Policies Agenda Project. Comparative Agendas Project. Disponível em http://www.comparativeagendas.net. Acesso em: 8 jul. 2016.

Borgatti, S.P. \& Everett, M.G., 1999. Models of Core/Periphery Structures. Social Networks, 21, pp.375-395. DOI: 10.1016/s0378-8733(99)00019-2

Borgatti, S.P. et al., 2009. Network Analysis in the Social Sciences. Science, 323(5916), pp.892-895. DOI: 10.1126/science. 1165821

Box-Steffensmeier, J.M. \& Christenson, D.P., 2014. The Evolution and Formation of Amicus Curiae Networks. Social Networks, 36, pp.82-96. DOI: 10.1016/j.socnet.2012.07.003

Degenne, A. \& Forsé, M., 1999. Introducing Social Networks. London: SAGE Publications.

Gould, R.V. \& Fernandez, R.M., 1989. Structures of Mediation: A Formal Approach to Brokerage in Transaction Networks. Sociological Methodology, 19, pp.89-126. DOI: 10.2307/270949

Hanneman, R.A. \& Riddle, M., 2005. Introduction to Social Network Methods. Riverside: University of California.

Heaney, M.T., 2014. Multiplex Networks and Interest Group Influence Reputation: An Exponential Random Graph Model. Social Networks, 36, pp.66-81. DOI: 10.1016/j.socnet.2012.11.003

Henning, C., 2009. Networks of Power in the CAP System of the EU-15 and EU-27. Journal of Public Policy, 29(2), pp.153-177. DOI: 10.1017/s0143814x09001056

Huckfeldt, R., 2009. Interdependence, Density Dependence, and Networks in Politics. American Politics Research, 37(5), pp.921-950. DOI: 10.1177/1532673x09337462

Janson, S. et al., 1993. The Birth of the Giant Component. Random Structures \& Algorithms, 4(3), pp.233-358. DOI: 10.1002/rsa.3240040303

Koger, G. \& Victor, J.N., 2009. The Beltway Network: A Network Analysis of Lobbyists’ Donations to Members of Congress. In American Political Science Meeting. Toronto.

Kolaczyk, E.D., 2010. Statistical Analysis of Network Data: Methods and Models. London: Springer.

Lapira, T.M.; Thomas, H.F. \& Baumgartner, F.R., 2009. The Structure and Stability of Lobbying Networks in Washington. In Annual Meeting of the Midwest Political Science Association. Chicago.

Lazer, D., 2011. Networks in Political Science: Back to the Future. PS: Political Science and Politics, 44(1), pp. 61. DOI: $10.1017 / \mathrm{s} 1049096510001873$

Lowery, D. et al., 2012. Generalist Interest Organizations and Interest System Density: A Test of the Competitive Exclusion Hypothesis. Social Science Quarterly, 93(1), pp.21-41. DOI: 10.1111/j.1540-6237.2011.00834.x

Marsden, P.V. \& Friedkin, N.E., 1993. Network Studies of Social Influence. Sociological Methods \& Research, 22(1), pp.127-151. DOI: 10.4135/9781452243528.n1

McClurg, S.D. \& Philips, J., 2011. A Social Network Analysis of Interest Group Contributions and Partisan Behavior in the 2006 House of Representatives (paper 26). In Political Network Summer Conference, Ann Arbor, MI. Disponível em: http://opensiuc.lib.siu.edu/cgi/viewcontent.cgi?article=1027\&context=pnconfs_2011. Acesso em: 28.ago.2016.

Pappi, F.U. \& Henning, C., 1998. Policy Networks: More than a Metaphor? Journal of Theoretical Politics, 10(4), pp.553-575. DOI: $10.1177 / 0951692898010004008$

, 1999. The Organization of Influence on the EC's Common Agriculturalpolicy: A Network Approach. European Journal of Political Research, 36(2), pp.257-281. DOI: 10.1111/1475-6765.00470

Santos, M.L.W.D., 2014. Representação de interesses na arena legislativa: os grupos de pressão na Câmara dos Deputados (1983-2012). Texto para discussão. Brasília: IPEA.

Schlozman, K.L., 2012. Counting the Voices in the Heavenly Chorus: Pressure Participants in Washington Politics. In The Scale of Interest Organization in Democratic Politics. Houndmills: Palgrave Macmillan.

Ward, M.D.; Stovel, K. \& Sacks, A., 2011. Network Analysis and Political Science. Annual Review of Political Science, 14, pp.245-264. DOI: 10.1146/annurev.polisci.12.040907.115949

Watts, D.J. \& Strogatz, S.H., 1998. Collective Dynamics of 'Small-World' Networks. Nature, 393(6684), pp.440-442. DOI: 10.1103/physreve.91.052815 


\title{
Networks of influence: mapping interest groups in the Brazilian National Congress
}

\begin{abstract}
The study aims to map the relations between interest groups in the Brazilian National Congress and to propose hypothesis about the population of interest groups. The guiding questions were: how interest groups articulate between themselves in the main political debates? Which are the most active groups? What are the main characteristics of the relationship structure? The data was collected from Congress websites. Every interest group participation in public hearings or seminars or similar events was registered for 2011 and 2012. This way, the study built a complete network with multi issue focus for all commissions of the Brazilian National Congress. The complete network has low density and several clusters around policy issues, making it closer to small world and a giant component models. Most organizations are are very specialized and is dedicated to only one policy. Nevertheless, a clear core with very central interests groups can be observed. The hypothesis is that interest groups that conforms the core have a special role and are more influente. In spite of that, none of the groups (or any coalition) seen to be predominant, as the centralization of the network is very low. The study presentes the first complete network of interest groups. The results indicate the existence of a core of organizations that exerts a structurally more important role. It is hypothesized that the centrality of interest groups can be a proxy for influence. From a theoretical perspective, it indicates the need to review pluralist thesis of atomization of interest groups.
\end{abstract}

KEYWORDS: interest groups; Brazilian National Congress; network analysis; lobby; decision-making.

This is an Open Access article distributed under the terms of the Creative Commons Attribution Non-Commercial License which permits unrestricted non-commercial use, distribution, and reproduction in any medium provided the original work is properly cited. 